\title{
VARIANCE STABILIZATION REVISITED: A CASE FOR ANALYSIS BASED ON DATA POOLING
}

\author{
A. M. FOWLER* \\ School of Geography, Geology and Environmental Science, The University of Auckland, Private Bag 92019, \\ Auckland, New Zealand
}

\begin{abstract}
The traditional approach to standardizing tree-ring time series is to divide raw ring widths by a fitted curve. Although the derived ratios are conceptually elegant and have a more homogenous variance through time than simple differences, residual heteroscedasticity associated with variance dependence on local mean ring width may remain. Incorrect inferences about climate forcing may result if this heteroscedasticity is not corrected for, or at least recognized (with appropriate caveats). A new variance stabilization method is proposed that specifically targets this source of heteroscedasticity. It is based on stabilizing the magnitude of differences from standardization curves to a common reference local mean ring width and uses data pooled from multiple radii. Application of the method to a multi-site kauri (Agathis australis (D. Don) Lindley) data set shows that (a) the heteroscedasticity issue addressed may be generic rather than radius-specific, at least for some species, (b) variance stabilization using pooled data works well for standardization curves of variable flexibility, (c) in the case of kauri, simple ratios do not appear to be significantly affected by this cause of heteroscedasticity, and (d) centennial-scale variance trends are highly sensitive to the analytical methods used to build tree-ring chronologies.
\end{abstract}

Keywords: heteroscedasticity, variance stabilization, kauri, Agathis australis.

\section{INTRODUCTION}

Fowler et al. (2008) summarized the potential of kauri (Agathis australis (D. Don) Lindley) as a proxy for the El Niño - Southern Oscillation (ENSO). They concluded that kauri has merit as an ENSO proxy within multi-proxy analyses (e.g. Braganza et al. 2009) but that the ENSO signal is not strong enough for stand-alone reconstruction of specific El Niño and La Niña years. However, they also concluded that the signal strength may be sufficient to allow the evolving variance of kauri tree-ring indices to be interpreted as a proxy for the evolving relative conjoint frequency of El Niño and La Niña events (ENSO robustness). Fowler (2008) tested this assertion, then exploited it to infer 423 years of ENSO robustness (Figure 1c, solid line), an analysis that suggests ENSO robustness has been characterized by a 55-80 year waxing and waning cycle (repeated seven times in

*E-mail: a.fowler@auckland.ac.nz the last 423 years) and by a centennial-scale increase to a $20^{\text {th }}$ Century peak.

The conclusion by Fowler (2008) that the $20^{\text {th }}$ Century may be the most active ENSO period is significant, especially in light of the known coincidence of late $20^{\text {th }}$ Century global warming and enhanced ENSO activity (e.g. Trenberth and Hoar 1996, 1997), but it is also controversial. For example, centennial-scale trends in variance could also be caused by non-stationarity of the teleconnection of ENSO to the north of New Zealand or to changing sensitivity of kauri tree rings to ENSO as trees age. It is also possible that trends in the evolving variance of tree-ring indices may be data-processing artifacts, introduced as a result of the standardization method typically used, as outlined below.

A 292-year sequence of tree-ring width measurements for one radius of a living kauri tree is shown in Figure 2. The superimposed smooth growth curve is a 20 -year spline $(50 \%$ frequency response at 20 years), fitted to the data series using 

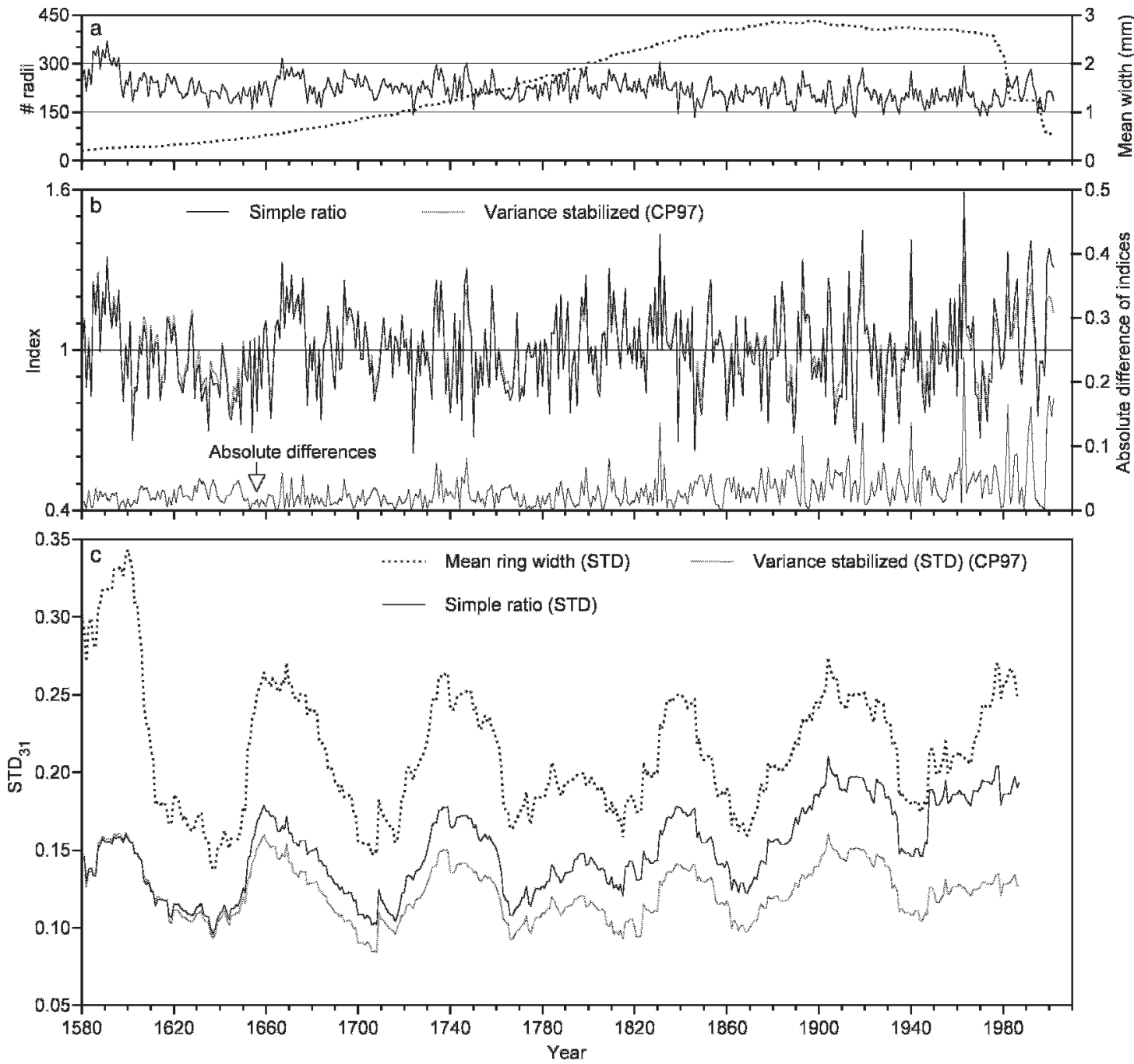

Figure 1. Evolutive variance of kauri master tree-ring chronologies. Panel a: mean ring width and sample depth (dotted line). Panel b: Simple ratio indices and variance stabilized indices using the Cook and Peters (1997) method, implemented in ARSTAN (Cook 1985), and absolute differences between the two time series. Panel c: running standard deviations for a 31-year window $\left(\mathrm{STD}_{31}\right)$ for the three tree-ring time series. Fowler et al. (2008) data set used.

ARSTAN (Cook 1985). The derived difference and ratio time series (Figures $2 \mathrm{~b}-\mathrm{c}$ ) both display systematic (and opposite) variance trends. Plotting the differences and ratios against the corresponding value for the fitted 20-year spline indicates strong dependence of the variance of the differences upon the local mean ring width (heteroscedasticity, Figure 2d) and a weaker reversed relationship in the case of the ratios (Figure 2e). This demonstrates that the trend in the variance of the differences (Figure 2b) is probably a conse- quence of reducing ring widths through time, and also, that calculating tree-ring indices using the traditional ratio method, in part aimed at producing a homoscedastic series (Fritts 1976), may only be a partial solution.

If the relationships apparent in Figure 2 are common across trees, then because declining mean ring width with tree age is typical, it is possible that the time-evolving variance of ratio tree-ring chronologies may be systematically biased. Specifically, periods of low (high) growth rate may have 


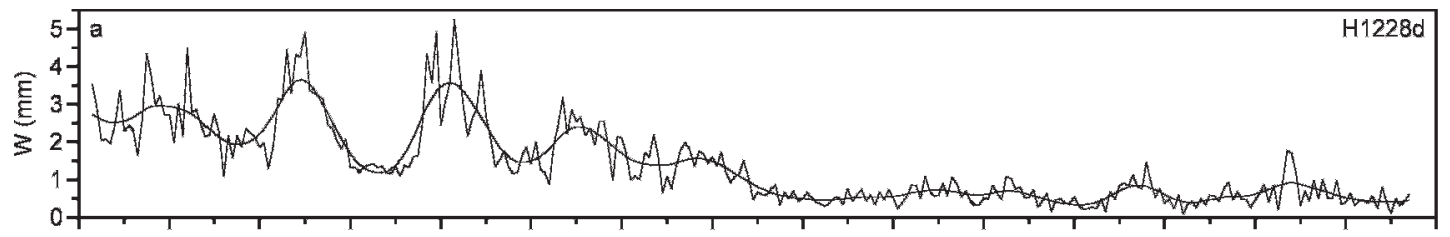

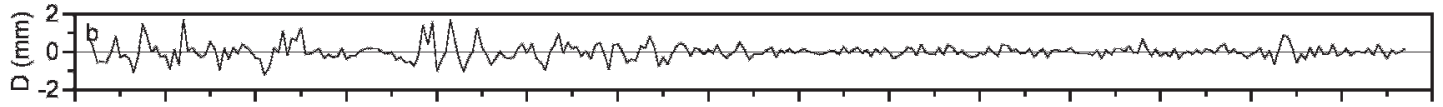
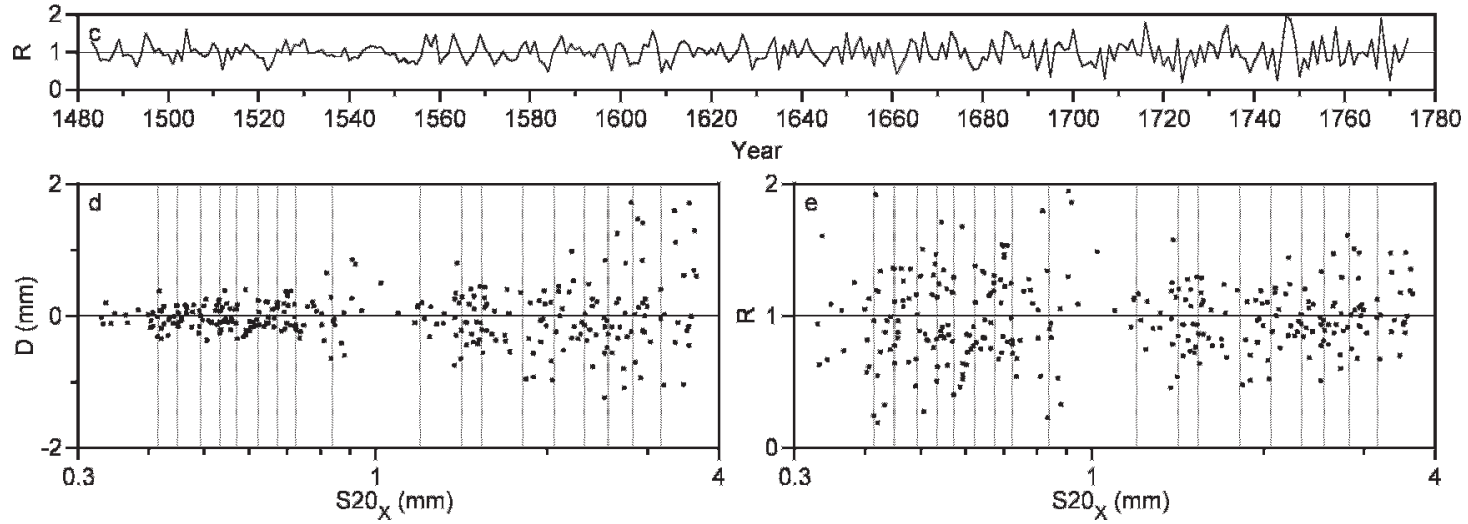

Figure 2. Heteroscedasticity of simple difference (D) and ratio (R) tree-ring indices. Panel a: ring widths (W) for H1228d with 20year fitted spline. Derived D and R time series are plotted as series (Panels b, c) and against their local values of the fitted spline $\left(\mathrm{S} 20_{\mathrm{X}}\right)$ (Panels d, e). Vertical dotted lines in the bottom panels split the data into 19 partitions (sorted on S20 $\mathrm{X}$ ) each of $15 / 16$ data points.

apparent high (low) variability that actually has nothing to do with climate forcing. Unless this potential bias has been shown to be insignificant or has been corrected for, climatic inferences based on the frequency of extremes or on local variance may be flawed. This is of specific concern in the kauri case because the upward trend in variance (Figure 1c) is associated with a decline in mean ring width (Figure 1a), suggesting that the variance trend may be an analytical artifact. Partitioning analysis of the pooled kauri data confirms that using ratios is efficient at removing much of the heteroscedasticity associated with differences, but also identifies residual dependence of ratios on the local mean ring width (Figure $3 \mathrm{a}-\mathrm{b}$ ). We can deduce from Figure $3 \mathrm{~b}$ that local variance will peak at a mean ring width of about $1.3 \mathrm{~mm}$ and will be systematically lower as mean ring width increases. It follows that some or all of the centennial-scale trend towards increasing local chronology variance is likely to be a data-processing artifact.

Cook and Peters (1997, hereafter CP97) addressed the issue of heteroscedasticity and proposed (p. 365) a "...data-adaptive power transformation method to stabilize the variance of ring-width series...", based on regression of the local spread of data on the local level. ("spread" and "level" are generic terms for data scatter [e.g. variance] and central tendency [e.g. mean], and "local" refers to statistics calculated for a specific year in a tree-ring chronology or for a few years straddling it.) The resulting regression equation is then used as the basis for transforming ring widths, prior to standardisation. Because of the transformation, ratios are no longer an appropriate statistic, so tree-ring indices are calculated as rescaled differences. CP97 recommended the abandonment of the ratio approach, in favour of variance stabilized residuals, but did note that the heteroscedasticity problems associated with ratios are not universal and that the transformation method “...can be excessive" (CP97, p. 369).

Application of the CP97 variance stabilization method to the kauri data produced a revised master chronology of rescaled residuals that is very similar to the simple ratios chronology 

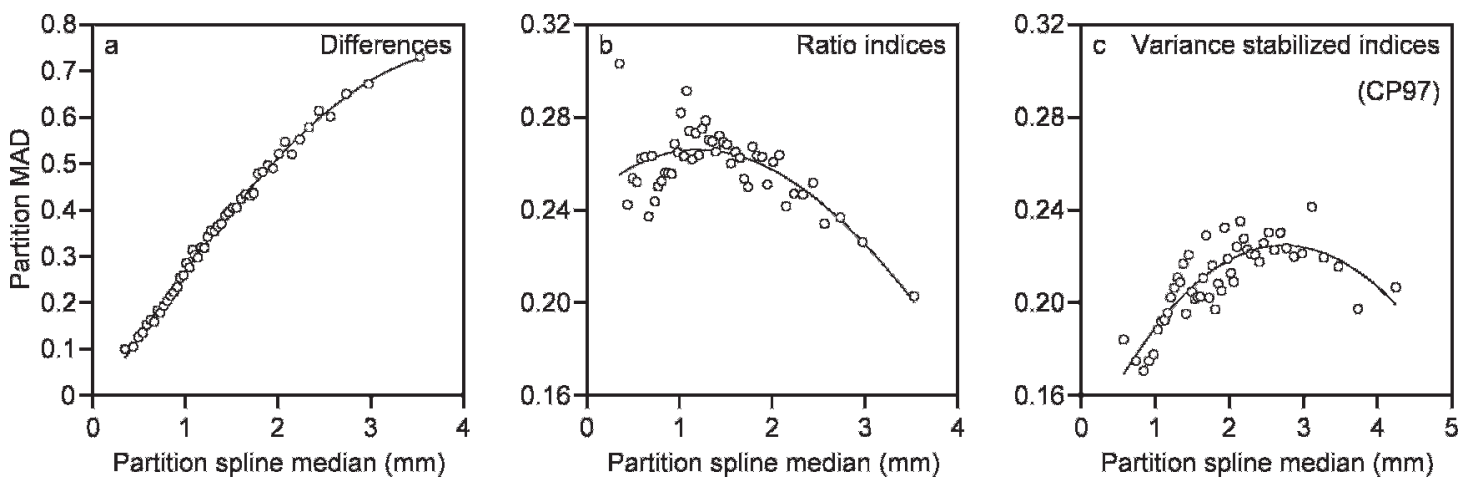

Figure 3. Dependence of the local "spread" of kauri tree-ring indices on the local "level", indicating residual bias in three chronologies (unbiased relationships would plot as horizontal lines). Analysis is based on the same data used in Figure 1 and each of the 50 plotted points represents a partition of 3506 rings drawn from multiple radii and sorted on the value of the fitted 200 -year spline. The spread of values for each partition is calculated using a robust measure of scatter (the median absolute difference from the median: MAD), which is plotted against the partition spline median. Fitted curves are third-order polynomials. Strong bias is evident in the differences (Panel a) and residual bias is also apparent for simple ratios (Panel b) and for variance stabilized indices (CP97, Panel c). Different x-axis scaling for the CP97 partitions is because they are calculated on transformed data.

(Figure 1b), yet sufficiently different in detail to significantly affect some aspects of the evolving variance (Figure 1c, thin dashed line). The aforementioned 55- to 80-year periodicity is retained, but centennial-scale trend has been almost completely eliminated, to the extent that the $20^{\text {th }}$ Century is no longer unusual in the context of the last four centuries. The direction of the latter change was expected, given the tendency towards reducing ring widths, however its scale was not, and some specific features are somewhat surprising (e.g. the magnitude of the down-scaling of the last of the seven variance peaks at the end of the $20^{\text {th }}$ Century). A close look at results for a number of individual radii indicated that the CP97 method generally performed well, but there were several instances where over-correction was apparent. This raises the possibility that the CP97 method may have introduced a "reversed heteroscedasticity" artifact into the kauri master (i.e. a real heteroscedasticity issue associated with simple ratios that has been over-corrected to give an opposite and equally undesirable heteroscedasticity problem). Partitioning analysis of variance stabilized indices strongly suggests that this is in fact the case, with clear evidence of residual dependence of the indices on local mean ring width (Figure 3c). The inference is that the kauri chronology derived using the CP97 variance stabilization method may be systematical- ly biased towards low variance when the local mean ring width is small.

The partitioning analysis results (Figure 3) show that the centennial-scale variance trends in kauri master chronologies may be exaggerated in the case of simple ratio indices but have been overcorrected (essentially removed) using the CP97 method. This prompted the revisiting of variance stabilization reported in this paper, with a view to deriving indices that do not carry a residual dependence on the local mean ring width (in this context, the "perfect" index would plot as a horizontal straight line in plots such as Figure $3 b-c)$. The method developed here is conceptually similar to CP97, in that the fundamental basis for variance stabilization is regression of the local "spread" of data against local "level". However, implementation details differ and a key underlying premise is different, in that it is assumed that the relationship between spread and level is not radius-specific and may well be obscured at the level of individual radii. It follows from this assumption that a pooled data approach may be appropriate, where relationships are derived using a much larger composite data set. Not withstanding this, for clarity the proposed method is first demonstrated using a single kauri radius. This is then expanded to explore the validity of data pooling at both site and multi- 

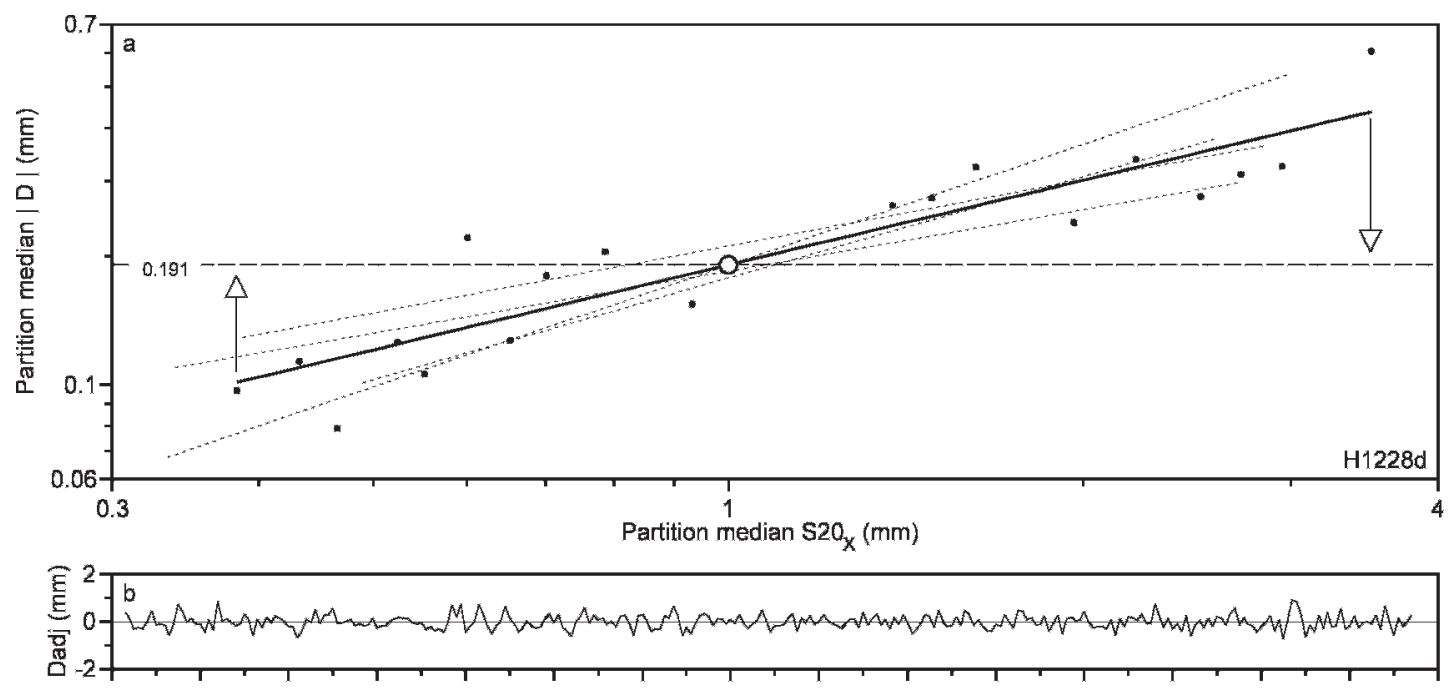

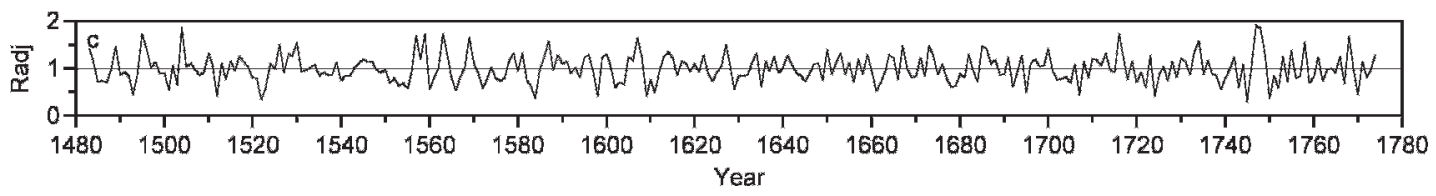

Figure 4. Radius-specific demonstration of the variance stabilization method (described in detail in the text). Panel a: partition medians $\left(S 20_{X},|D|\right)$ from Figure $2 d$ (dots). The thick line is the regression of $|\mathrm{D}|$ on $\mathrm{S} 20_{\mathrm{X}}$. Thin dashed lines are equivalent results for four other radii from the same tree (data not shown). The open circle on the regression line represents the reference point $\left(\mathrm{S} 20_{\text {ref }}=\right.$ $1.0 \mathrm{~mm}$ ), which has an estimated $|\mathrm{D}|$ of $0.191 \mathrm{~mm}$. Arrows show the rotation through this hinge point needed to stabilize variance (scaling for other values of $S 20_{X}$ to bring $|D|$ close to 0.191). Panels b, c: resulting variance-stabilized $D\left(D_{\text {adj }}\right)$ and $R\left(R_{a d j}\right)$ time series (compare with Figure 2b, c).

site levels. Again for clarity, the method is initially demonstrated using standardization curves that are much more flexible than those typically used for climate reconstruction purposes. A test of how well the method scales to more conservative standardization concludes the analysis. The analyses mostly use the kauri data set from Huapai Scientific Reserve, presented in detail in Fowler and Boswijk (2001) and Fowler et al. (2005). All additional data used are described in Fowler et al. (2004) and Fowler et al. (2008).

\section{VARIANCE STABILISATION METHOD AT RADIUS LEVEL}

Vertical dotted lines in Figure 2d partition the 292 difference (D) values for H1228d into 19 subsets of 15 or 16 data points, sorted on the corresponding value for the fitted 20-year spline $\left(\mathrm{S} 20_{\mathrm{X}}\right)$, and Figure $4 \mathrm{a}$ shows the relationship between $|\mathrm{D}|$ and $\mathrm{S} 20_{\mathrm{X}}$ for the medians of these data partitions. In effect, the data shown in Figure $2 d$ have been "folded" on the $\mathrm{D}=0$ line by changing all negative values to positives (reasonable because the symmetrical "cone" pattern was found to be similar across multiple radii investigated). Then the medians of $\mathrm{S} 20_{\mathrm{X}}$ and $|\mathrm{D}|$ were calculated for each partition. Partitioning was done to tease out the underlying bias, by eliminating the inter-annual scatter associated with climate forcing, and also to reduce the influence of outliers. Experimentation showed that a partition size of about 15 data points are sufficient for this purpose and that bigger partitions can be counter-productive (for individual radii) because of the associated reduction in the number of partitions. However, little benefit seemed to gained from having more than about 30 partitions, so for larger data sets the partition size was allowed to increase.

An estimate of the dependence of $|\mathrm{D}|$ on $\mathrm{S} 20_{\mathrm{X}}$ is provided by the linear regression equation:

$$
\log _{\mathrm{e}}|\mathrm{D}|=\mathrm{a}_{\log } \mathrm{S} 20_{\mathrm{X}}+\mathrm{b},
$$


which can be rewritten as:

$$
|\mathrm{D}|=\mathrm{S} 20 \mathrm{x}^{\mathrm{a}} \mathrm{e}^{\mathrm{b}}
$$

where $\mathrm{a}$ and $\mathrm{b}$ are regression coefficients and $\mathrm{e}$ is the base of natural logarithms.

Two values for exponent " $a$ " in Equation 2 are important in the context of variance stabilization. First, if the exponent is zero, then $|\mathrm{D}|$ equals a constant $\left(\mathrm{e}^{\mathrm{b}}\right)$. This would mean that $|\mathrm{D}|$ is independent of $\mathrm{S} 20_{\mathrm{X}}$ and therefore the $\mathrm{D}$ time series can reasonably be assumed to have no heteroscedasticity associated with evolving mean ring widths. Second, if the exponent equals one, Equation 2 simplifies to a linear regression equation with zero intercept. In this case the D time series would be heteroscedastic, but because $\mathrm{D}$ is proportional to $\mathrm{S} 20_{\mathrm{X}}$, the ratios should be homoscedastic. It follows that we should be able to stabilize the variance by scaling the data to target an exponent of zero or one (respectively for homoscedastic differences or ratios). For consistency with CP97, the former approach is taken here.

Targeting a value of zero for exponent " $a$ " in Equation 2 is analogous to rotating the solid regression line in Figure 4a clockwise through a selected pivot reference point. For pragmatic reasons (see below) $\mathrm{S} 20_{\mathrm{X}}=1 \mathrm{~mm}$ was selected to define the $\mathrm{x}$-axis value for this reference point $\left(\mathrm{S} 20_{\text {ref }}\right)$ with the $\mathrm{y}$-axis value (here 0.191$)$ obtained from the regression line equation. Implementation of this scheme involved calculating "adjusted" D $\left(D_{\text {adj }}\right)$ by multiplying $D$ by the ratio of the regression estimate of $|\mathrm{D}|$ for $\mathrm{S} 20_{\text {ref }}$ to the equivalent estimate for $\mathrm{S} 20_{\mathrm{X}}$ (corresponding to the specific D). Thus:

$$
\mathrm{D}_{\mathrm{adj}}=\mathrm{D}\left(|\mathrm{D}|_{\mathrm{regR}} /|\mathrm{D}|_{\mathrm{regX}}\right)
$$

where $|\mathrm{D}|_{\text {regR }}$ is the regression estimate of $|\mathrm{D}|$ for $\mathrm{S} 20_{\text {ref }}$, and $|\mathrm{D}|_{\mathrm{regX}}$ is the regression estimate of $|\mathrm{D}|$ for $\mathrm{S} 20_{\mathrm{X}}$.

The net effect of the transformation was to scale $\mathrm{D}$ by $0.43\left(\mathrm{~S} 20_{\mathrm{X}}=3.65 \mathrm{~mm}\right)$ to $2.08\left(\mathrm{~S} 20_{\mathrm{X}}=\right.$ $0.33 \mathrm{~mm}$ ). Visual comparison of the $\mathrm{D}$ and $\mathrm{D}_{\text {adj }}$ series (Figures 2b, 4b) suggests that Equation 3 is indeed an effective solution to the heteroscedasticity of the former. Just how effective is apparent from comparison of the cumulative frequency distributions of the first and last 100 years of the time series, before and after adjustment (Figure $5 \mathrm{a}, \mathrm{b})$. Whereas the $\mathrm{D}$ plots are notably divergent, those for $\mathrm{D}_{\text {adj }}$ are similar. Because the 20-year spline fitted growth curve has been removed, $\mathrm{D}_{\text {adj }}$ is a (more) homoscedastic standardized tree-ring chronology.

Because $\mathrm{D}_{\text {adj }}$ is D scaled to $\mathrm{S} 20_{\text {ref, }}$, it seems reasonable to calculate an equivalent adjusted $\mathrm{R}$ $\left(\mathrm{R}_{\mathrm{adj}}\right)$ by simply adding $\mathrm{S} 20_{\text {ref }}$ to $\mathrm{D}_{\mathrm{adj}}$, then dividing by $\mathrm{S} 20_{\text {ref: }}$ :

$$
\begin{gathered}
\mathrm{R}_{\mathrm{adj}}=\left(\mathrm{S} 20_{\text {ref }}+\mathrm{D}_{\mathrm{adj}}\right) / \mathrm{S} 20_{\text {ref }} \\
\mathrm{R}_{\mathrm{adj}}=1.0+\mathrm{D}_{\mathrm{adj}} / \mathrm{S} 20_{\text {ref }}
\end{gathered}
$$

and, because $\mathrm{S} 20_{\text {ref }}$ here is $1.0 \mathrm{~mm}$, this further simplifies to:

$$
\mathrm{R}_{\mathrm{adj}}=\mathrm{D}_{\mathrm{adj}}+1.0
$$

resulting in an identical cumulative frequency plot to $\mathrm{D}_{\mathrm{adj}}$, apart from the $\mathrm{x}$-axis values (Figures $5 \mathrm{~b}, \mathrm{~d}$ ). The only benefit of this trivial transformation is that $R_{\text {adj }}$ values can be interpreted, and compared to other series, with the conceptual elegance associated with ratios (see discussion in CP97). Results for an equivalent analysis of H1228d using the variance stabilization approach of CP97 (implemented in ARSTAN) show comparable success in stabilizing the variance (Figures 5e-f), although a slight over-correction is apparent, particularly at the high end.

The generic applicability of the method described above was tested by applying it to all 94 radii from the Huapai site. For multi-centennial series, the results are broadly similar to the patterns shown in Figure 2 and Figure 4, with sufficient spread in the value for the local mean to establish meaningful relationships with $|\mathrm{D}|$, similar to Figure $4 \mathrm{a}$. The only significant issue arising relates to missing rings. Because the homoscedasticity adjustments are based on transformations of D, true missing rings may not have an $\mathrm{R}_{\mathrm{adj}}$ of zero, and it is possible for non-missing rings to end up with negative $R_{\text {adj. }}$. The former is reasonable if missing rings are more common when growth is suppressed, as is the case for kauri. The latter was dealt with by 

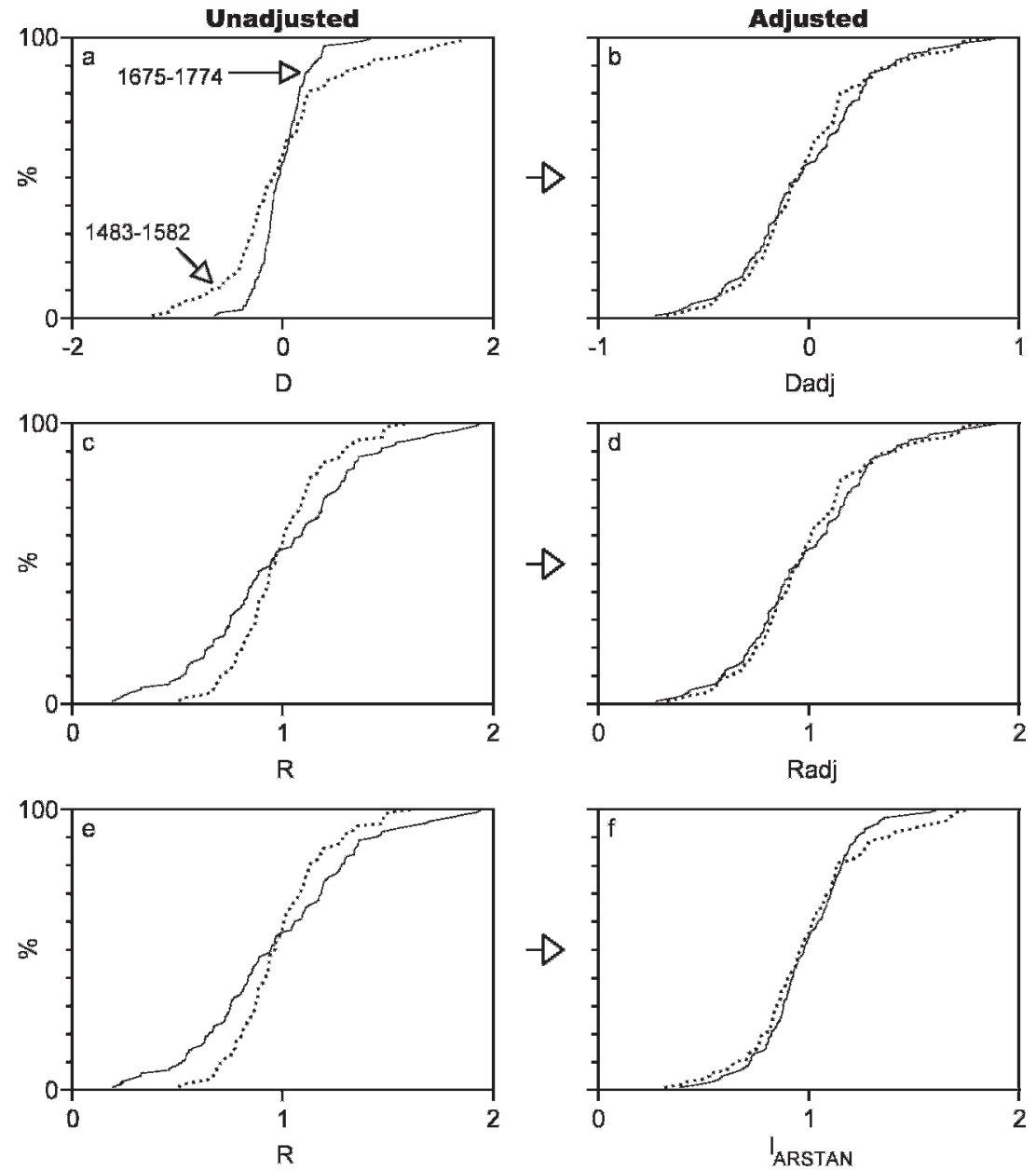

Figure 5. Impact of variance stabilization adjustments for H1228d. Panels a, c: cumulative frequency distributions of D and R for the first and last 100 years of Figure 2b, c. Panels b, d: equivalent results for the variance-stabilized series $\left(D_{\text {adj }}, R_{\text {adj }}\right)$ from Figure 4b, c. Panel e: same as Panel c (and almost identical), but calculated by ARSTAN. Panel f: equivalent analysis of ARSTANderived indices using the data-adaptive power-transform option.

simply imposing a lower $\mathrm{R}_{\text {adj }}$ limit of zero. A total of 30 rings from 13 radii needed to be adjusted.

For series less than about 200 years long, radius-specific application of the method was less convincing. Relationships between $|\mathrm{D}|$ and $\mathrm{S} 20_{\mathrm{X}}$ were generally weaker and often notably divergent from the general pattern. This was particularly the case when series were too short to provide a reasonable $\mathrm{S} 20_{\mathrm{X}}$ range (i.e. notably less than the order of magnitude range shown for $\mathrm{H} 1228 \mathrm{~d}$ in Figure 4a). To reduce the influence of such (assumed) spurious relationships, minimum (0.2) and maximum (5.0) D-scaling limits were intro- duced based on pooled regression analysis (next section). A total of 46 rings from nine radii were affected by these limits, 33 of which were from three radii.

\section{DATA POOLING}

The relationships between $|\mathrm{D}|$ and $\mathrm{S} 20_{\mathrm{X}}$ for each of the 94 Huapai radii are shown in Figure $6 a$, with Figures $6 b$ and $6 c$ showing the associated frequency distributions for "a" and " $\mathrm{e}$ " " (from Equation 2). Note that the median value for the exponent "a" $(0.915)$ is close to the 

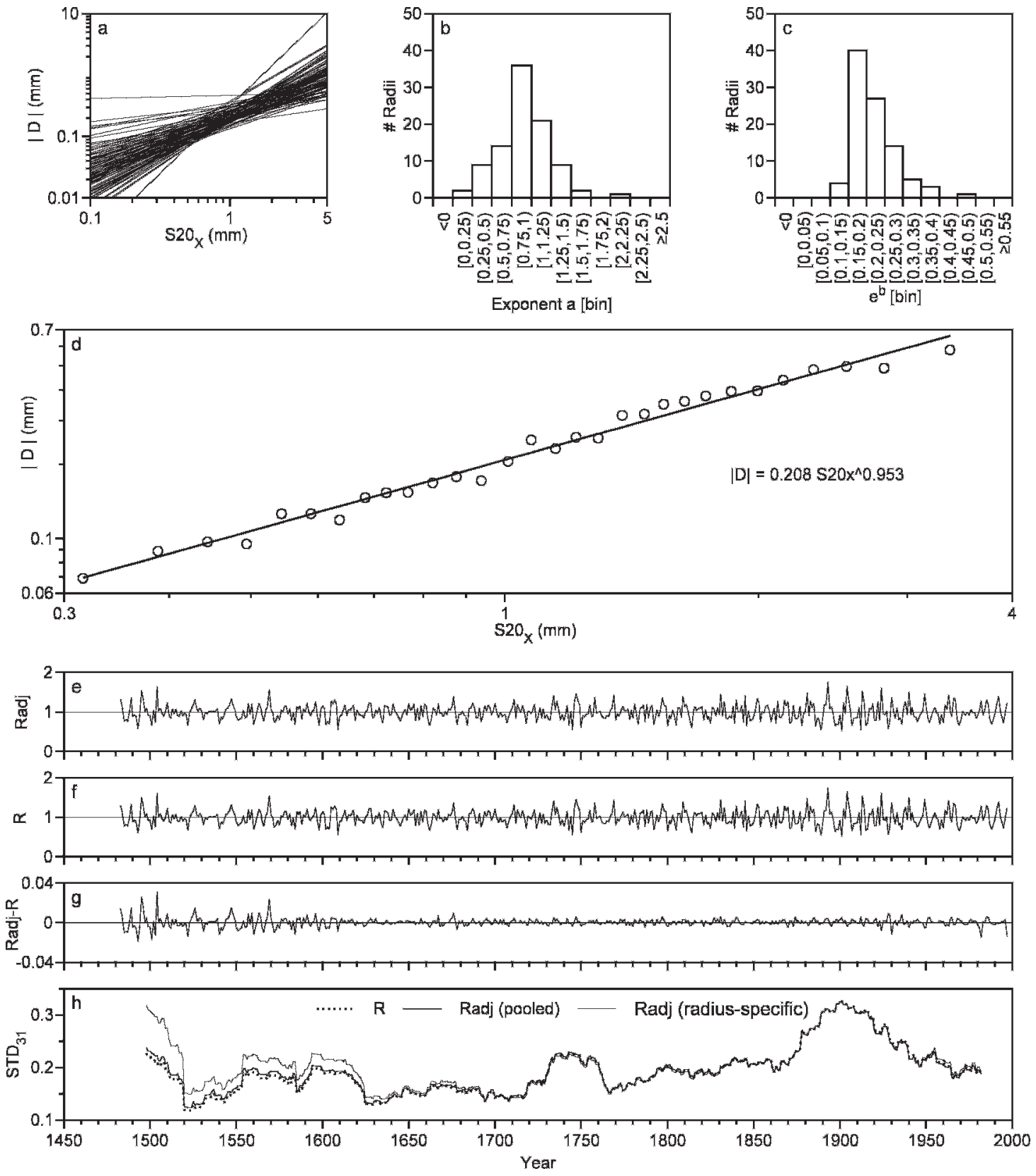

Figure 6. Application of the variance stabilization method to 94 radii from one site (Huapai Scientific Reserve). Panel a: regression lines equivalent to that shown for H1228d in Figure 4a. Panels b, c: frequency distributions for regression equation terms "a" and "e $\mathrm{b}$ " (Equation 2). Panel d: pooled-data relationship (each circle represents 718 observations drawn from many, but not necessarily all, 95 radii). Panel e: $R_{\text {adj }}$ site chronology, derived by averaging the $94 \mathrm{R}_{\text {adj }}$ series, each variance-stabilized using the pooled-data relationship (Panel d, Equations 3-6). Panel f: simple R site chronology (no variance stabilization). Panel g: difference between the $\mathrm{R}_{\mathrm{adj}}$ and R chronologies. Panel h: 31-year running standard deviation ( $\mathrm{STD}_{31}$ ) through $\mathrm{R}$ and $\mathrm{R}_{\text {adj. }}$ " $\mathrm{R}_{\text {adj }}$ (pooled)" is equivalent to $R_{\text {adj }}$ in Panel e. " $R_{\text {adj }}$ (radius-specific)" refers to an additional site chronology (not shown) built using radius-specific application of the variance stabilization method. 
critical value of one, where the power equation simplifies to a linear regression of $|\mathrm{D}|$ on $\mathrm{S} 20_{\mathrm{X}}$ with zero intercept, $|\mathrm{D}|$ becomes simply a scaled (by $\mathrm{e}^{\mathrm{b}}$ ) function of $\mathrm{S} 20_{\mathrm{X}}$, and $\mathrm{R}$ and $\mathrm{R}_{\text {adj }}$ are identical. Values close to one are typically associated with radii where ratio series (i.e. $\mathrm{R}$ ) are essentially homoscedastic. That the Huapai median is close to one indicates that the heteroscedasticity of $\mathrm{R}$ evident in Figures $2 \mathrm{c}$ and $2 \mathrm{e}$ is not a generic feature of kauri at this site, although the fact that $65 \%$ of radii have values less than one does indicate a weak tendency in that direction.

The data for all 94 radii were pooled and the relationship between $|\mathrm{D}|$ and $\mathrm{S} 20_{\mathrm{X}}$ recalculated (Figure 6d) for the combined data set (30 partitions each of about 718 rings). $R_{\text {adj }}$ was then computed for each radius using this equation and the resulting series averaged to produce an $R_{\text {adj }}$ site chronology (Figure 6e). Because the transformation equation exponent "a" $(0.953)$ is again close to one, the derived $\mathrm{R}_{\text {adj }}$ series is very similar to the simple $\mathrm{R}$ series (Figures $6 \mathrm{f}-\mathrm{g}$ ). Consequently, 31-year running standard deviations (STD) are also practically identical (Figure 6h), the only discernable difference being a very small increase in $\mathrm{R}_{\mathrm{adj}}$ (pooled) STD prior to 1600 .

Also plotted in Figure 6h (thin line) is the corresponding running STD plot for an alternative $R_{\text {adj }}$ site chronology, where the variance of each radius time series was stabilized using radiusspecific relationships (Figure 6a). Two interesting features emerge. First, post-1650 ( $>20$ radii), the three STD series are essentially identical. Although radius-specific relationships between $|\mathrm{D}|$ and $\mathrm{S} 20_{\mathrm{X}}$ differ markedly, these tend to cancel out as sample size increases, converging to the pooleddata relationship. Second, there is a notable divergence in the plots pre-1600, which increases as sample depth declines. This is a consequence of the few radii spanning this period (e.g. reduced to 5 radii from 3 trees at 1550) having relationships between $|\mathrm{D}|$ and $\mathrm{S} 20_{\mathrm{X}}$ similar to H1228d (Figure 4a) and relatively wide rings over the period, resulting in relatively large differences between $\mathrm{R}_{\mathrm{adj}}$ (radius-specific) and $\mathrm{R}$ (compare the first 100 years in Figures 2c and 4c). It is plausible that the radius-specific analysis is closest to reality and that resorting to a pooled relationship has resulted in a loss of information. However, it is also possible that we are seeing an artifact caused by low sample depth. Subsequent analysis (below) suggests that the latter is the more likely explanation.

The analysis reported above for Huapai was repeated for three other kauri chronology sites (Cascades, Trounson, Manaia Sanctuary) with very similar results. For each site, values for exponent "a" in Equation 2 straddle (but are mostly less than) one and pooled-data analysis consistently produced $R_{a d j}$ series very similar to uncorrected R. Differences between the two series are larger than those shown for Huapai (Figure $6 \mathrm{~g}$ ) by up to a factor of about two, but running STD plots for $\mathrm{R}$ and $\mathrm{R}_{\mathrm{adj}}$ are very similar within each site. Similar to Huapai, the Manaia Sanctuary plots are almost identical, but both Cascades and Trounson have slightly higher variance in the $16^{\text {th }}$ and early $17^{\text {th }}$ Centuries following transformation. None of the three additional sites displays the radius-specific analysis anomaly noted for Huapai.

The pooled-data regression equations for the four sites exhibit strong agreement (Figure 7), suggesting that the relationship between $|\mathrm{D}|$ and $\mathrm{S} 20_{\mathrm{X}}$ can reasonably be treated as generic and data therefore pooled from across kauri's growth range. Accordingly, the analysis was rerun on the composite multi-site 527 radii data set of Fowler et al. (2008), producing the multi-site relationship identified by the plotted partition data (circles) and thick regression line in Figure 7. The relationships shown here were the basis for establishing the D-scaling bounds noted at the end of the previous section. Fewer than $1 \%$ of rings have associated $\mathrm{S} 20_{\mathrm{X}}$ values less than 0.2 or greater than $5 \mathrm{~mm}$, and for this range the scaling factors calculated from the equations shown in Figure 7 are enveloped by 0.22 and 4.6. These limits were relaxed to the 0.2 to $5.0 \mathrm{D}$-scaling bounds previously noted. Applying the multi-site relationship to all radii, 50 rings $(<0.05 \%)$ from 8 radii $(1.5 \%)$ had scaling limits applied and 13 rings from 11 radii had negative $R_{\text {adj }}$ changed to zero. These figures are about an order of magnitude less than the number of adjustments required for radius-specific application of the method (777 


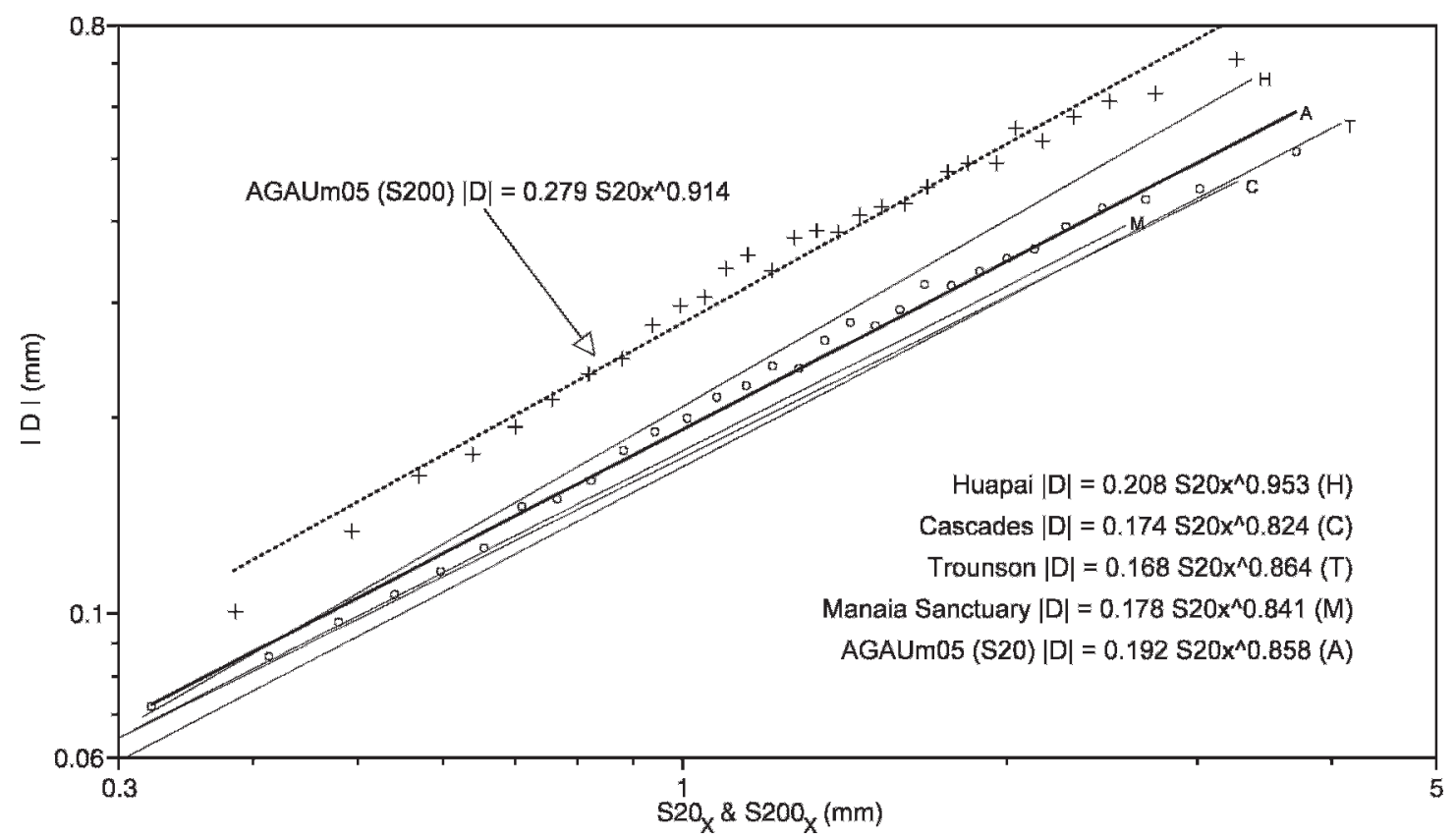

Figure 7. Comparison of pooled-data relationships between $|\mathrm{D}|$ and $\mathrm{S} 20_{\mathrm{X}}$ for four sites $(\mathrm{H}, \mathrm{C}, \mathrm{T}, \mathrm{M})$ and across multiple sites (A). The regression line for Huapai $(\mathrm{H})$ is identical to Figure $6 \mathrm{~d}$. Circles are partition medians for regression on all data. The dashed line and "+" symbols are also for regression on all data, but using more conservative standardization (see Section "Multi-decadal and centennial trends").

rings from 44 radii for scaling and 401 rings from 89 radii for zero adjustment).

Running-STD plots for three kauri master chronologies built using the simple ratio method and for the pooled and radius-specific application of the variance stabilization method are compared in Figure 8b. The fact that the respective time series are nearly identical is a key result, implying that the heteroscedasticity apparent in simple ratio-based kauri tree-ring indices at the radius level (e.g. Figure 2c) does not flow through to significant systematic bias at the master chronology level. Coupled with this, it is also apparent that pooled and radius-specific analyses give comparable results. This was somewhat surprising given the much larger number of "corrections" needed (application of scaling limits and changing negative $\mathrm{R}_{\mathrm{adj}}$ to zero) in the radius-specific case. It is also noteworthy that the differences between the two methods identified for Huapai in Figure 6h (1500-1650) are absent in Figure 8. This suggests that the earlier result was probably an artifact of low sample depth and points to a potential problem of radius-specific application of the method when sample depth is low.

Low sample depth also probably explains the slightly less consistent fit between the three running-STD series from the late- $13^{\text {th }}$ through late- $15^{\text {th }}$ Centuries (Figure 8b). However, it cannot explain the minor divergence of the radiusspecific $\mathrm{R}_{\mathrm{adj}}$ running-STD after 1750 , because sample depth peaks at about 1900 . This divergence is probably caused by a combination of changing sample mix and subtle site-specific differences in the relationship between $|\mathrm{D}|$ and $\mathrm{S} 20_{\mathrm{X}}$. Specifically, Huapai is the dominant site through this period. In terms of sample depth (Fowler et al. 2004), it has relatively high site chronology STD over the relevant period (Figure 6h), and the STD of most Huapai radii is slightly underestimated using the pooled data equation.

The time series plotted in Figure 8 extend 300 years earlier than in Figure 1, into periods of much lower sample depth. Recall that the variance stabilization method proposed here is intended to remove bias associated with the dependence of 


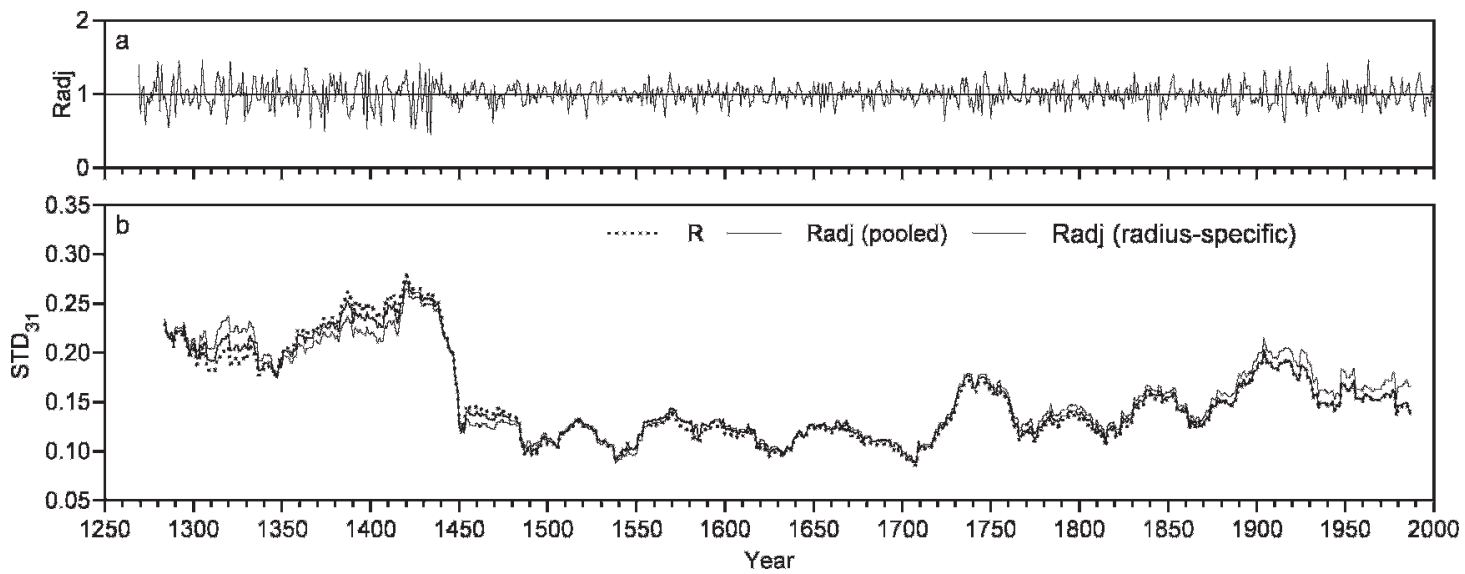

Figure 8. Impact of variance stabilization on the evolutive variance of kauri master tree-ring chronologies. Panel a: Kauri master chronology built from 527 radii from multiple sites (one high altitude site excluded). 20-year standardization splines were used for all radii and variance was stabilized based on the pooled data relationship shown in Figure 7 (regression line A). Panel b: 31-year running standard deviation $\left(\mathrm{STD}_{31}\right)$ through a simple ratio chronology $(\mathrm{R})$ and two $\mathrm{R}_{\text {adj }}$ chronologies built using pooled and radiusspecific application of the variance stabilization method. Note that only the $R_{\text {adj }}$ master chronology for pooled-data variance stabilization is shown because the three series are practically indistinguishable.

variance on the local mean. Other sources of heteroscedasticity are unaffected, most notably variance inflation as sample size becomes small (Osborn et al. 1997). This partly explains the higher $\mathrm{R}_{\text {adj }}$ variance prior to 1450 (Figure 8b).

\section{MULTI-DECADAL AND CENTENNIAL TRENDS}

Very flexible smoothing splines were used in the previous sections to develop and demonstrate the variance stabilisation method. However, in the context of most dendroclimatological applications, the resulting time series would have limited value, because the derived $R_{\text {adj }}$ series lack multidecadal and greater variance. This section explores whether the method is also applicable in the case of more conservative standardisation curves that preserve multi-decadal to centennial-scale variance.

The results of replicating the analyses shown in Figures 2 and 4, using a stiffer 200-year smoothing spline $(50 \%$ variance response at 200 years), are shown in Figure 9. Because the fitted curve declines through almost its entire length, the relationships of $\mathrm{D}, \mathrm{R}$, and $|\mathrm{D}|$ to $\mathrm{S} 200_{\mathrm{X}}$ (Figure 9d-f) all carry strong echoes of the decadal-scale variability of the original series.
For example, the persistently negative values for D near $\mathrm{S} 200_{\mathrm{X}}=1.0 \mathrm{~mm}$ are associated with a $c a$. 30 -year period in the mid-1600s, when ring widths are less than the fitted curve. This flows through to $\mathrm{R}$ and to the $|\mathrm{D}|$ partitions, resulting in a weaker relationship between $|\mathrm{D}|$ and $\mathrm{S} 200_{\mathrm{X}}$ than was the case for the 20-year spline analysis. Still, the overall relationship for $\mathrm{H} 1228 \mathrm{~d}$ is reasonably well defined and both $\mathrm{D}_{\text {adj }}$ and $\mathrm{R}_{\mathrm{adj}}$ are less heteroscedastic than D and R (Figure 10a-d).

Comparing Figure 10c with Figure 5c, it is apparent that the "reversed" heteroscedasticity of $\mathrm{R}$ identified in the 20-year spline analysis is less of an issue in the case of the more conservative spline. As a consequence, although applying the variance stabilization method still results in some improvement (Figure 10d), this is modest. Interestingly, the CP97 variance stabilization method implemented in ARSTAN actually seems to make the heteroscedasticity of H1228d indices worse. Whereas the simple ratios have higher variance in the latter part of the series (Figure 10e), this is reversed and amplified following transformation.

Radius-specific application of the method across the full kauri data set proved problematic. Weaker relationships between $|\mathrm{D}|$ and $\mathrm{S} 200_{\mathrm{X}}$ amplified problems previously identified in the 20-year spline analysis. Specifically, D-scaling 

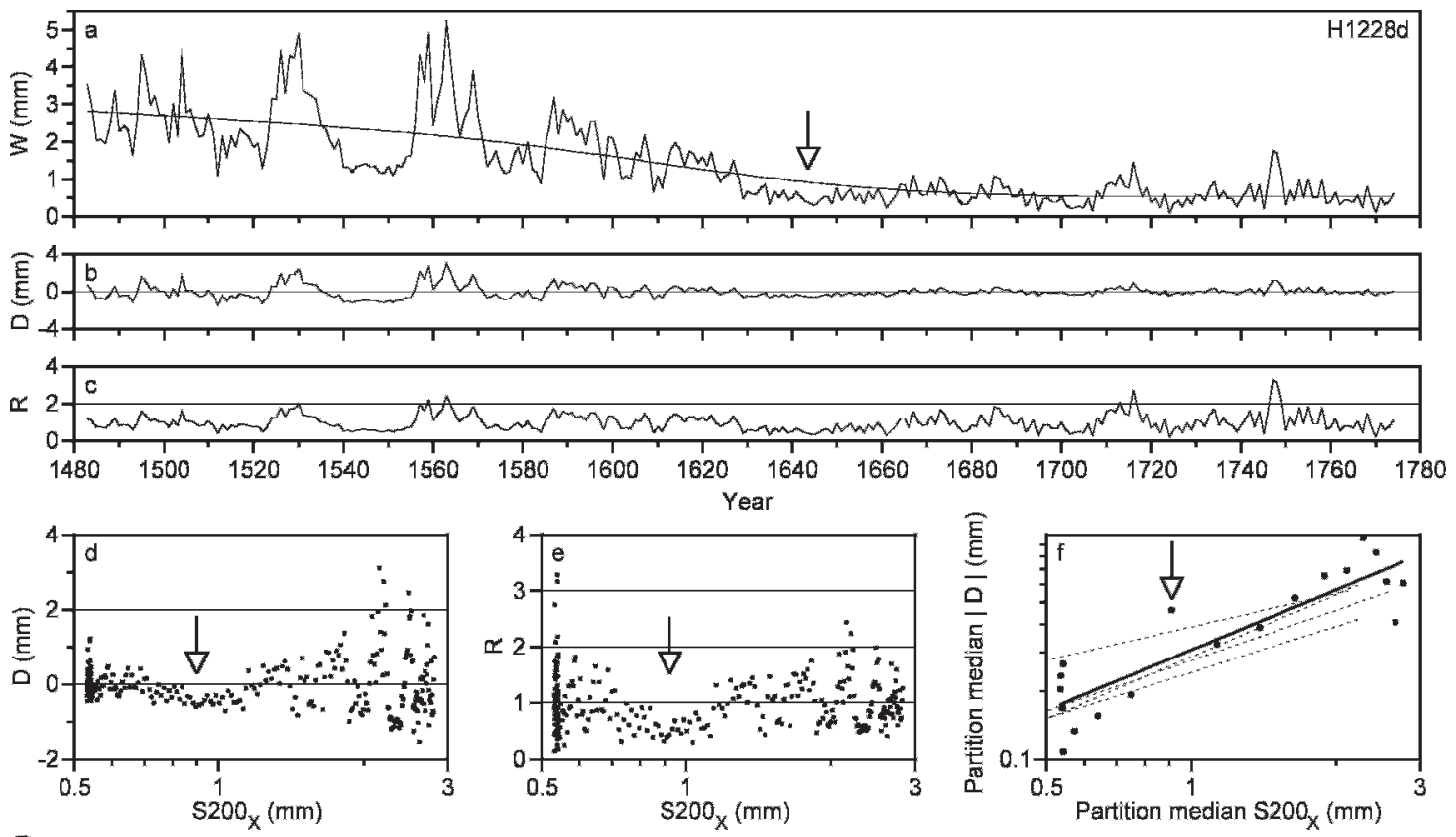

焉 ${ }_{0}^{4}$

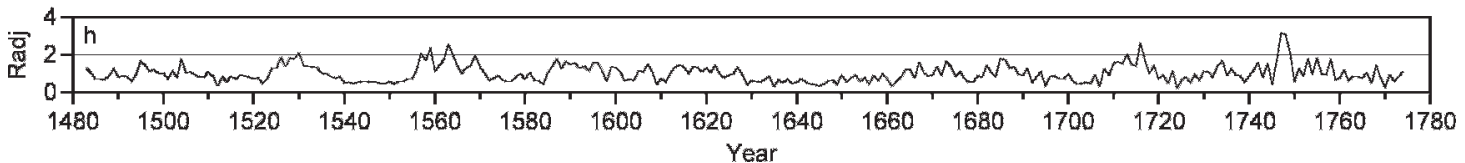

Figure 9. Same as combined Figure 2 and Figure 4, for a 200-year smoothing spline. Arrows identify the consequence of systematic departure of $\mathrm{W}$ from the fitted curve (see text).

$\left(|\mathrm{D}|_{\text {regR }} /|\mathrm{D}|_{\text {regX }}\right.$ in Equation 3) needed to be choked back to the 0.2 to 5.0 permitted range for 4,300 rings from 58 radii (previously 46 rings from 9 radii) and 2,122 rings from 173 radii had negative $\mathrm{R}_{\mathrm{adj}}$ adjusted to zero (previously 30 from 13 ). This number of corrections was considered too great to warrant pursuing radius-specific application further.

The pooled-data analysis results are much more encouraging. Because many radii contribute to each data partition of about 3,506 rings, radiusspecific problems associated with decadal-scale influences largely disappear, and the underlying relationship emerges clearly (Figure 7, top regression line). The relationship between $|\mathrm{D}|$ and $\mathrm{S} 200_{\mathrm{X}}$ is marginally weaker than that derived using 20year splines $\left(\mathrm{R}^{2}=0.98\right.$ vs. 0.99), and the slightly steeper regression line is displaced (because $|\mathrm{D}|$ decreases with more flexible fitted splines). Deriving $\mathrm{R}_{\mathrm{adj}}$ using the pooled-data relationship, 55 rings $(0.05 \%)$ from 11 radii $(2 \%)$ had $D$-scaling choked back to the 0.2 and 5.0 bounds, and a further 55 rings from 28 radii $(5 \%)$ had negative values of $R_{a d j}$ changed to zero.

A pooled-data master $R_{\text {adj }}$ chronology built using the procedure previously described is almost identical to the simple ratio chronology $\left(\mathrm{R}^{2}=\right.$ 0.9995) and the respective running 31-year STD plots reveal only trivial differences (Figure 11). Part of the reason for the similarity is apparent when the dependence of $R_{\text {adj }}$ spread on local level is compared to the equivalent results for simple ratios (Figure 12a). In essence, the original relationship (dashed line) has been rotated anticlockwise through a partition spline median of about $1.0 \mathrm{~mm}$. This has removed the trend 

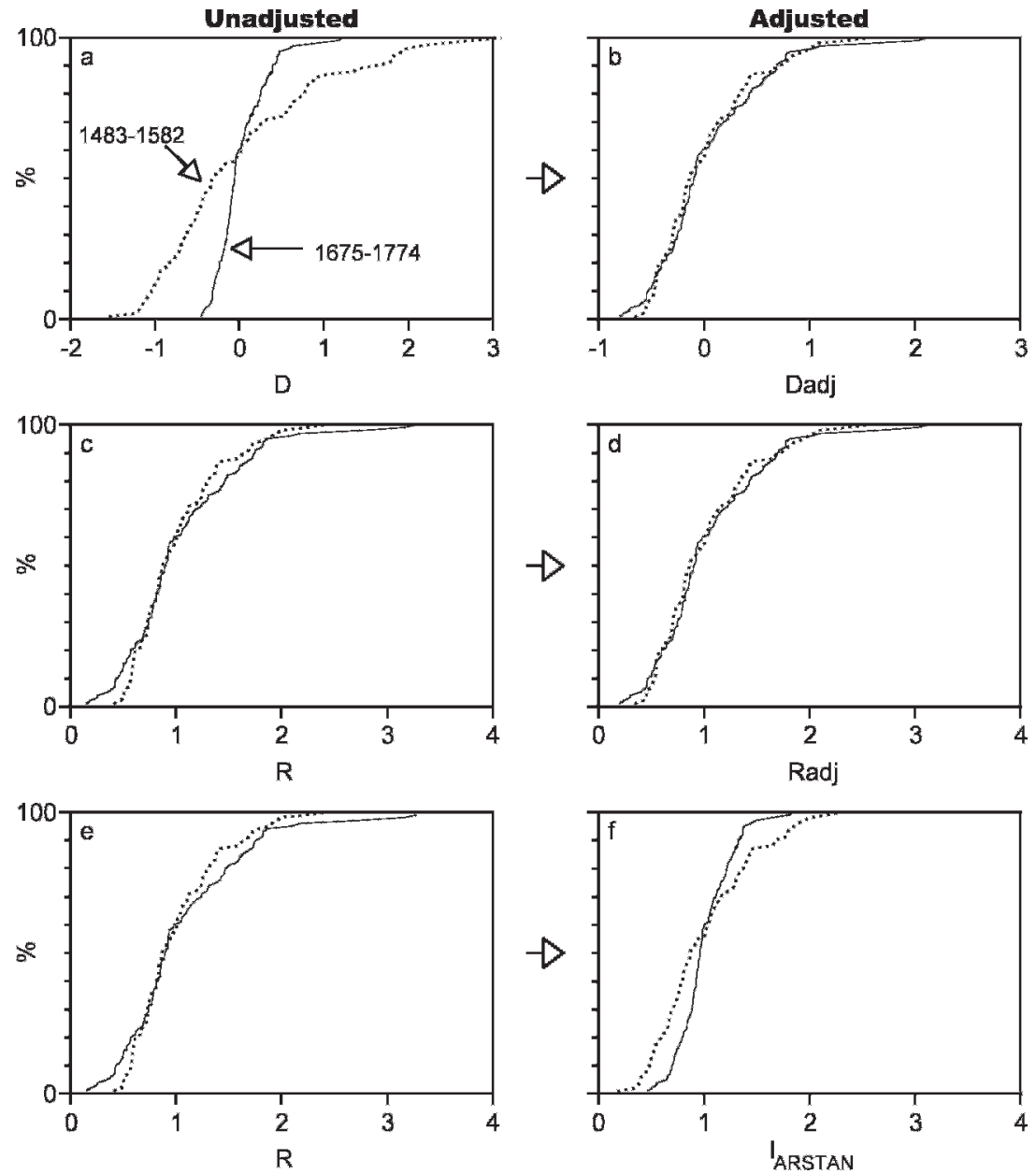

Figure 10. Same as Figure 5, for a 200-year smoothing spline.

towards lower variance as local mean ring widths become larger, but retained the relationship's distinctive bow shape. Two other pertinent features to note are the fact that the relationship lines "cross", allowing cancelling out of effects (see below), and the small scale of the differences, especially near typical mean ring widths (1.0$2.0 \mathrm{~mm}$, Figure 1a). Also important is the large spread of values for the fitted splines (e.g. Figure $12 b-c)$, relative to the subdued changes in mean ring width (Figure 1a). The inter-relationships between these factors are complex and best explained by example.

Consider the 1830 ring. Calculated over 362 radii, local mean ring width $(1.46 \mathrm{~mm})$ is large, relative to most of the $19^{\text {th }}$ and $20^{\text {th }}$ Centuries, and
$76 \%$ of fitted spline curves have values greater than $1.0 \mathrm{~mm}$ (Figure 12b), above which the $\mathrm{R}_{\mathrm{adj}}$ relationship consistently plots above that for simple ratios (Figure 12a). However, the resulting increase in variance is small, partly because most radii are clustered in the $1.0-2.0 \mathrm{~mm}$ range where the difference between the $R_{\text {adj }}$ and simple ratio relationships is minor, and partly because the $24 \%$ of radii with fitted spline values less than $1.0 \mathrm{~mm}$ have an opposing influence (because the relationships reverse). The net effect is a $0.0025(1.7 \%)$ increase in STD for the 31 years centred on 1830 for the $\mathrm{R}_{\mathrm{adj}}$ chronology, relative to the simple ratio chronology. Mean ring width generally declines from 1830 to about $0.9 \mathrm{~mm}$ in 1970 (Figure 1a) and the proportion of radii with fitted spline curve 


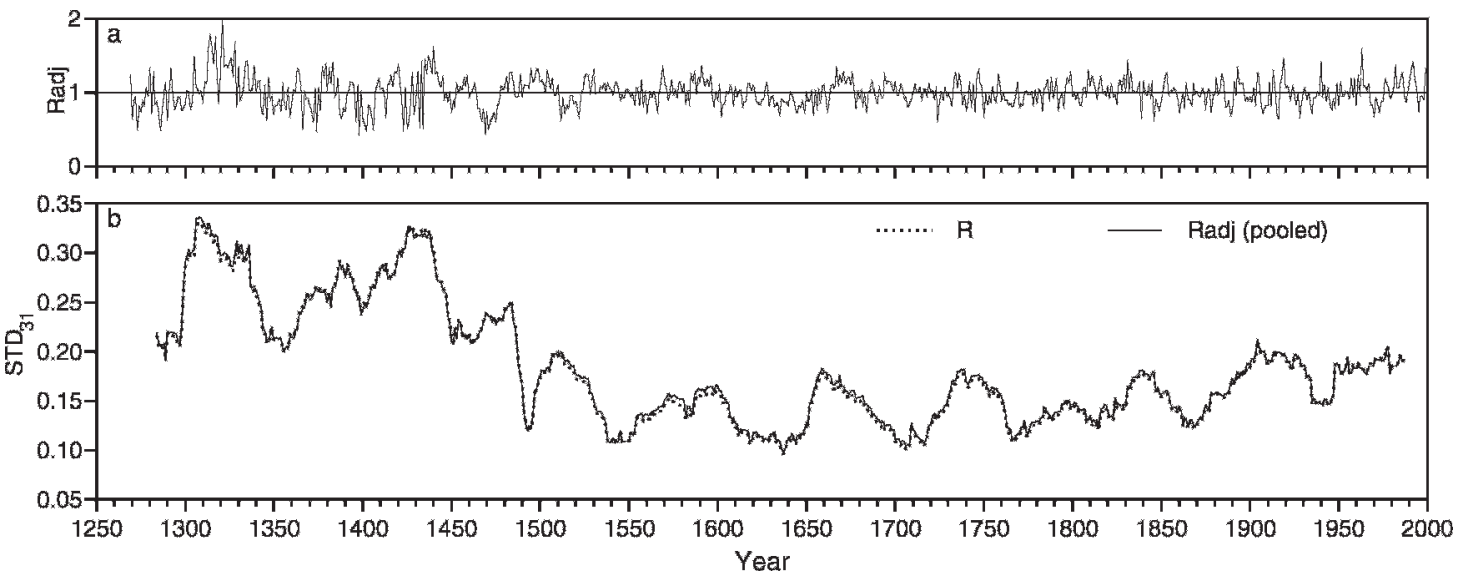

Figure 11. Same as Figure 8, but using 200-year smoothing splines and absent the radius-specific analysis running STD.

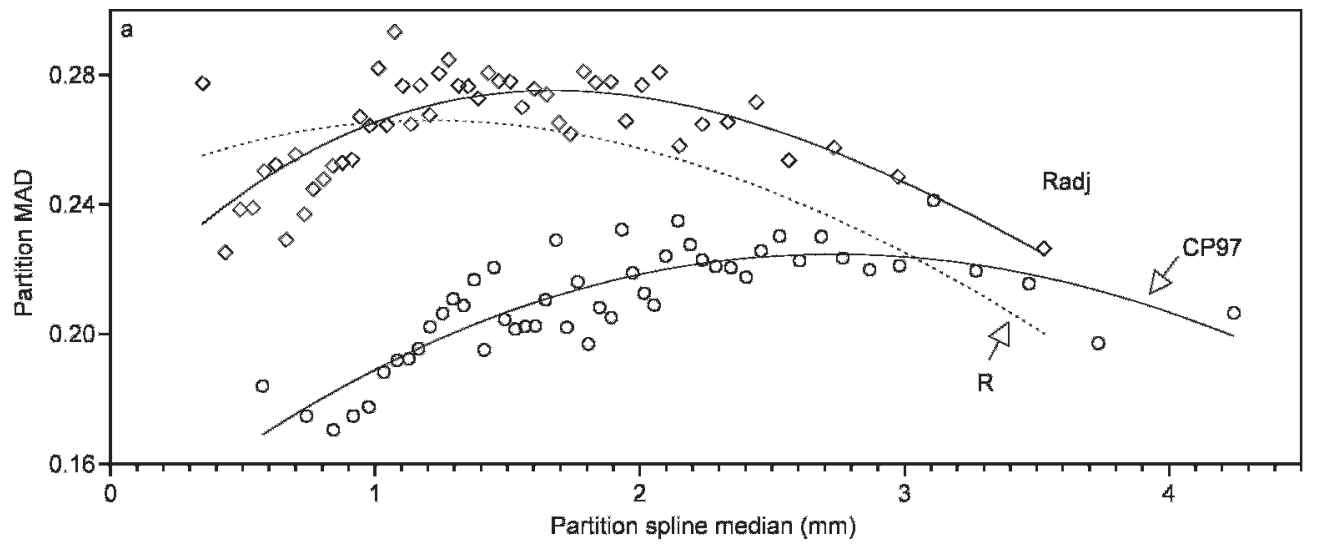

b 1830

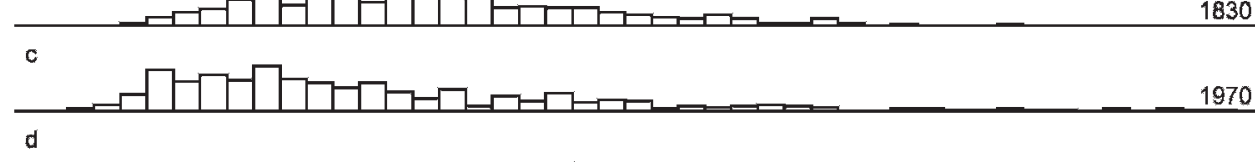
\}raw

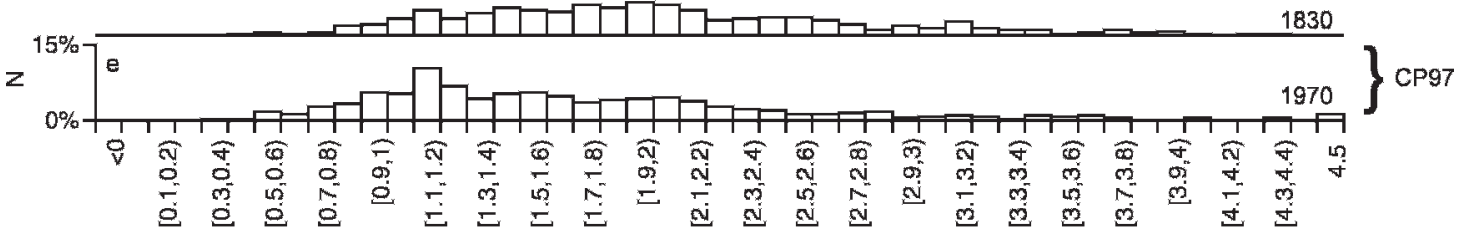

Bin

Figure 12. Residual bias of the local "spread" (MAD) of kauri tree-ring indices on the local "level" (partition spline median) in three tree-ring chronologies. Panel a: Relationships for R and CP97 are the same as those shown in Figure 3b, c (data points for R not shown for clarity). $R_{\text {adj }}$ is variance stabilized using the method developed here. Fitted curves are third-order polynomials. Panels b-e: frequency distributions of values for the fitted 200-year splines for a wide (1830) and a narrow (1970) ring. Note that the CP97 transformed data are shifted right (Panels d, e). 
values less than $1.0 \mathrm{~mm}$ increases to $50 \%$ (Figure 12c). The running STD plots (Figure 11b) cross at 1946, and by 1970 the difference is of similar magnitude $(-0.0023)$ to 1830 . This trend with declining mean ring width is as expected, but the scale of changes is universally minor.

\section{DISCUSSION AND CONCLUSIONS}

This paper has presented an alternative method for correcting tree-ring chronologies for heteroscedasticity associated with dependence of the local variance on the local mean ring width (represented here by the fitted standardization curve). The core of the approach is to scale $\mathrm{D}$ to a common reference value for the local mean (here $1.0 \mathrm{~mm})$, based on regressing $|\mathrm{D}|$ on the local mean ring width, for partitioned data. Partitioning is central to the method, needed to resolve the underlying bias, which is small relative to interannual variability, and also to reduce the influence of outliers. The derived $D_{\text {adj }}$ time series is then converted to the traditional ratio index form by adding in, then dividing by, the local mean reference value.

Radius-specific application of the method worked well when the standardization curve was flexible (i.e. the 20-year spline used here) and the local mean ring widths varied by about an order of magnitude. Problems were sometimes encountered when the latter condition was not met, because of insufficient data range to adequately resolve relationships, requiring the scaling range to be somewhat arbitrarily bounded ( 0.2 to 5.0 here). Also, when converting to ratios, it is possible to compute a negative $\mathrm{R}_{\text {adj }}$ value, requiring application of a zero limit. These problems were markedly amplified when a more conservative standardization curve (200-year spline) was used, to the extent that radius-specific application was considered inappropriate.

Relationships between $|\mathrm{D}|$ and $\mathrm{S} 20_{\mathrm{X}}$ for the 94 radii from the Huapai Scientific Reserve chronology site were found to be consistent in form, suggesting an underlying relationship that is not radius-specific. The fact that separate site chronologies built using radius-specific and pooled-data relationships are essentially identical when sample depth was $20+$ radii demonstrates the validity of data pooling. Moreover, subsequent analyses indicated that the divergence in the chronologies over the first 100 years (Figure 6h) is probably a low sample depth artifact. Comparison of pooled-data site relationships (Figure 7) also showed strong consistency, suggesting that pooling data from across kauri's growth range is reasonable. Again, the validity of doing so is confirmed by the very close agreement between kauri master chronologies computed from 527 radii from multiple sites using both radius-specific and pooled-data relationships (Figure 8b). In contrast to the radius-specific application of the method, pooled-data application scaled well to use with more conservative standardization techniques.

The methodology presented here combines standardization and variance stabilization. This is because $D_{\text {adj }}$ and $R_{\text {adj }}$ both derive from the detrended difference series (i.e. D). One reason for pursuing this conjoint approach was the opportunity it seemed to provide for deriving variance-stabilized ratios. The idea here was that, because $\mathrm{D}_{\text {adj }}$ is stabilized to some reference mean ring width, that reference value could be used to compute variance-stabilized ratios (Equations 46). Unfortunately, experimentation with different reference mean ring widths (not shown) indicated that $R_{\text {adj }}$ is sensitive to the reference value chosen. Although the results for kauri are encouraging, that may be a fortuitous consequence of the selected reference value being very close to kauri's mean ring width. Moreover, such conjoint treatment may be undesirable, because it circumvents opportunities to apply alternative analytical procedures, such as the auto-regressive modelling capability provided by ARSTAN. Intuitively it makes sense to separate data pre-processing from standardization, if possible (discussed further below).

The key driver for the research presented here was concern that centennial-scale trend in the variance of kauri master chronologies derived using the simple ratio method (Figure 1c) may be an analytical artifact, related to trends in mean ring width. A convincing result emerging from this research is that this is not so. The heteroscedas- 
ticity of ratios evident for H1228d (Figures 2c, 5c) is relatively small and, more importantly, is not ubiquitous. The $\mathrm{R}$ and $\mathrm{R}_{\mathrm{adj}}$ chronologies built for sites and for composite masters are almost identical and the running variance plots are not meaningfully different (Figures 6, 8, 11). This indicates that, in the case of kauri, ratios do adequately correct for the heteroscedasticity associated with the dependence of the variance of differences with local mean ring width. Other sources of heteroscedasticity remain, including the elevated variance pre-1500 caused by low sample depth, but these are outside the scope of this paper.

The benign conclusion above presupposes that the variance stabilization method developed here is correct and, by implication, that the CP97 method has over-corrected to the extent that "reversed heteroscedasticity" has been introduced. This is most clearly evident in the residual bias revealed by partitioning analysis (Figure 12), which shows increasing spread (partition MAD) as mean ring width increases. The increasing divergence between the $\mathrm{CP} 97$ and simple ratio relationships as mean ring widths decrease (Figure 12) explains the divergence of the respective running STD plots as mean ring widths decline over the last 400 years (Figure 1c). We can reasonably infer that this difference between the two methods is not a consequence of data pooling because radius-specific and pooled-data application of the proposed method give nearly identical results (e.g. Figure 8b). What may be telling though is that the CP97 over-correction seems to increase as the standardization becomes more conservative ( $c f$. Figure 5f, 10f), which brings into focus an important point of difference between the two methods.

In essence, the variance stabilization method presented here is based on pooled differences between raw ring widths and fitted standardization splines, and the results vary with selected spline stiffness. In contrast, the CP97 method stabilizes the variance of first-order differences around a tightly-fitted running mean and is independent of standardization. The CP97 method is conceptually most similar to the flexible spline application of the new method (Figure 2), and is similarly effective when a flexible spline is used for standardization ( $c f$. Figure 5d, f). However, if a stiff standardization spline is used, decadal-scale peaks and troughs become more important, disproportionately contributing to both large negative and large positive differences about the standardization spline. The first half of the series in Figure 9a is an example of such a situation, resulting in the previously-noted CP97 method over-correction (Figure 10f). If, as is common, peaks and troughs are larger in absolute terms as mean ring width increases, it follows that tree-ring indices derived from differences (rather than ratios) will tend to have a local spread that is positively biased as local mean ring width increases. This is consistent with the positive trend in the CP97 residual bias analysis (Figure 12).

An implication I draw from the above is that variance stabilization should take account of the stiffness of the standardization curves used (as done with the method proposed here). On the other hand, there is obvious merit in the CP97 approach of dealing with variance stabilization prior to standardization. If data pooling is acceptable, then a possible way forward would be to stabilize the variance prior to standardization using a fitted spline with a frequency response that is consistent with the intended standardization. The stabilization could use the approach proposed here or a variant of the CP97 method, but data pooling would be a prerequisite.

Finally, a key generic finding of this research is that centennial-scale variance trends in tree-ring chronologies are highly sensitive to the analytical method used to minimize residual heteroscedasticity associated with the dependence of local variance on local mean ring width (and other sources of heteroscedasticity not considered here). This implies the need for considerable caution in interpreting such trends, including statements concerning the relative frequency of wide and narrow rings, which implicitly assume that any trends are real. It would be wise in any case to test for residual bias in the relationship between local spread and the local mean and to compare this to trends in mean ring width. Partitioning analysis (e.g. Figure 12) is a simple yet effective tool for doing this. 


\section{ACKNOWLEDGMENTS}

Financial support for this research was provided by the New Zealand Foundation for Research, Science and Technology (Contract UOAX0714). The quality of this paper was much improved by the considered comments of two anonymous reviewers.

\section{REFERENCES CITED}

Braganza, K., J. L. Gergis, S. B. Power, J. S. Risbey, and A. M. Fowler, 2009. A multi-proxy index of the El Niño-Southern Oscillation, A.D. 1525-1982. Journal of Geophysical Research 114, D05106, doi: 10.1029/2008JD010896.

Cook, E. R., 1985. A Time Series Analysis Approach to Tree Ring Standardization. Ph.D. dissertation, The University of Arizona, Tucson.

Cook, E. R., and K. Peters, 1997. Calculating unbiased tree-ring indices for the study of climatic and environmental change. The Holocene 7:359-368.

Fowler, A., and G. Boswijk, 2001. Tree-ring analysis of kauri (Agathis australis) from Huapai Scientific Reserve, Huapai, Auckland. New Zealand Tree-Ring Site Report No. 6, Department of Geography Working Paper No. 13, The University of Auckland.
Fowler, A. M., 2008. ENSO history recorded in Agathis australis (kauri) tree rings. Part B: 423 years of ENSO robustness. International Journal of Climatology 28:2135.

Fowler, A. M., G. Boswijk, J. Gergis, and A. Lorrey, 2008. ENSO history recorded in Agathis australis (kauri) tree rings. Part A: Kauri's potential as an ENSO proxy. International Journal of Climatology 28:1-20.

Fowler, A. M., G. Boswijk, and J. Ogden, 2004. Tree-ring studies on Agathis australis (kauri): A synthesis of development work on Late Holocene chronologies. Tree-Ring Research 60:15-29.

Fowler, A., A. Lorrey A, and P. Crossley, 2005. Seasonal growth characteristics of kauri. Tree-Ring Research 61:3-20.

Fritts, H. C., 1976. Tree Rings and Climate. Academic Press, London.

Osborn, T. J., K. R. Briffa, and P. D. Jones, 1997. Adjusting variance for sample-size in tree-ring chronologies and other regional mean time series. Dendrochronologia 15:89-99.

Trenberth, K. E., and T. J. Hoar, 1996. The 1990-1995 El NiñoSouthern Oscillation event: Longest on record. Geophysical Research Letters 23:57-60.

, 1997. El Niño and climate change. Geophysical Research Letters 24:3057-3060.

Received 3 March 2008; accepted 27 February 2009. 